\title{
A New Discrete Tomographic Reconstruction Method for Electron Tomography
}

\author{
Ilke Arslan ${ }^{1}$, Toby Sanders ${ }^{1,2}$, Peter Binev ${ }^{2}$, Bruce C. Gates ${ }^{3}$, Alexander Katz ${ }^{4}$ \\ ${ }^{1}$ Pacific Northwest National Laboratory, Richland, WA 99352, USA. \\ ${ }^{2}$ University of South Carolina, Columbia, SC, 29208, USA \\ ${ }^{3}$ University of California-Davis, Davis, CA 95616, USA. \\ ${ }^{4}$ University of California-Berkeley, Berkeley, CA 94720, USA.
}

We have developed a new and advanced reconstruction algorithm for electron tomography data using a combination of compressed sensing and discrete reconstruction methods. Our algorithm not only provides accurate reconstructions with limited projection images, but also with a limited tilt range. This algorithm offers a solution to the decades-old "missing wedge" artifact present in reconstructions with limited data. With the ability to provide high quality reconstructions with only $\sim 30$ images as opposed to the standard $\sim 120$, this algorithm opens the door to materials characterization of electron beam sensitive materials across all fields of science. This 3-D characterization method with $1 \mathrm{~nm}$ to sub-nm resolution provides a new methodology for the advancement of nanotechnology.

The reduced number of images necessary for a robust 3-D reconstruction also opens the door for 3-D chemical imaging, using EDX or EELS, where the number of tilts at which data are acquired is also limited. Standard methods of 3D reconstruction, such as weighted back projection (WBP) and simultaneous iterative reconstruction technique (SIRT), are not equipped to handle this lack of information, and result in significant blurring. Two of the recent successful algorithms are the discrete algebraic reconstruction technique (DART) and total variation (TV) minimization within compressed sensing (CS). The DART algorithm uses an algebraic reconstruction method (ARM) and pairs it with the prior knowledge that there are only a small number (two or three) of different materials in the sample, each corresponding to a different gray value in the reconstruction. An initial reconstruction is computed using the ARM and rounded to the chosen fixed gray values based on some threshold, and iteratively refined using the ARM. The method of TV minimization stems from the mathematical theory of compressed sensing and only recently became available due to new computational methods for solving the TV minimization problem. The method considers the characterization of real images and encourages the reconstruction to take larger jumps in gray values to create clear boundaries, hence creating a similar effect to that of DART.

Figures 1-2 show a comparison of using DART with SIRT as an initial solution, and TVM as an initial solution. Using all of the projections for this data set (73 images), the two solutions are quite similar (Figure 1). However, using half of the data (37 images), it can be seen in Figure 2 that SIRT+DART does not reconstruct the small particles within the pores, while TVM+DART is able to reconstruct the particles. Further examples of these methods will be presented, covering different material geometries, such as layered zeolite materials, porous supports, and particles in supports.

This research was funded in part by the laboratory directed research and development (LDRD) program at the Pacific Northwest National Laboratory, and in part by the DOE BES DE-SC0005822. PNNL is operated by Battelle under contract DE-AC05-76RL01830. 

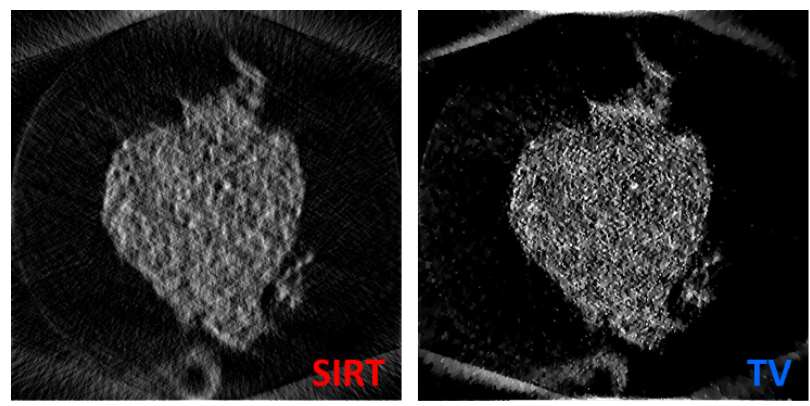

\section{Projections}
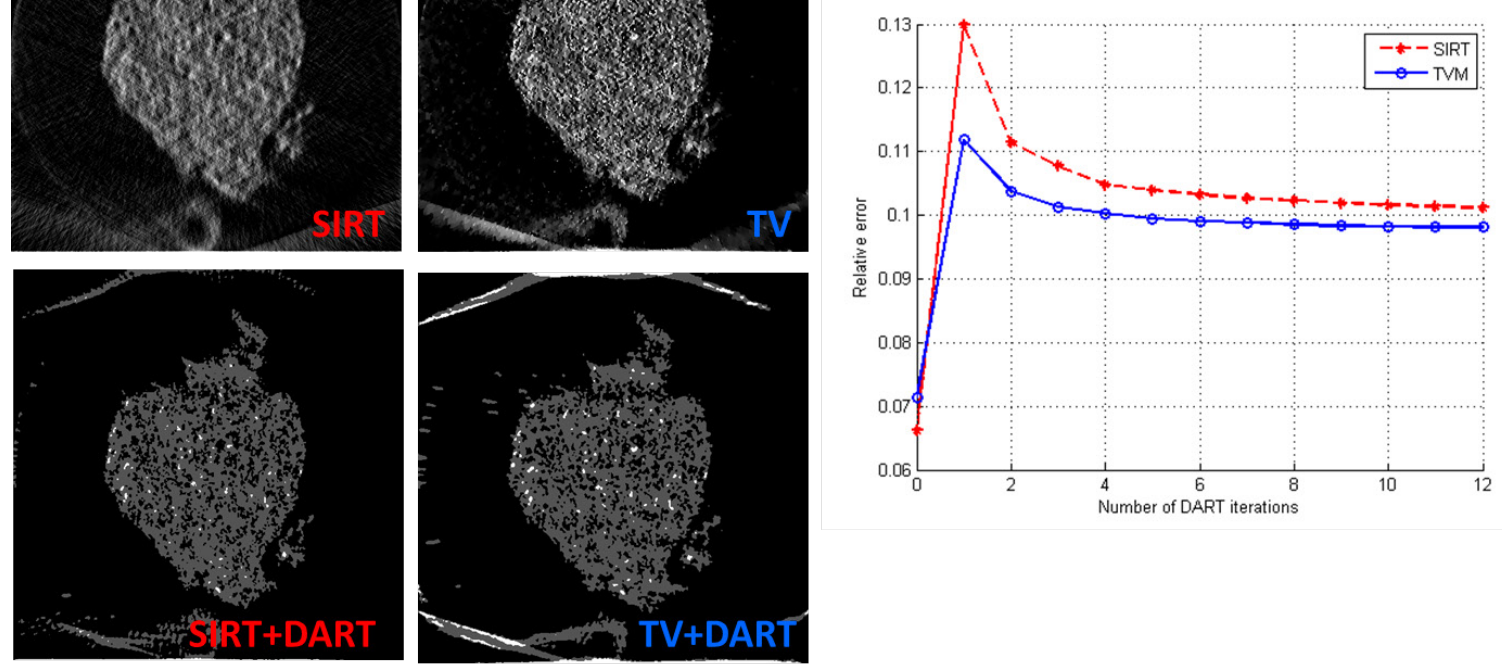

Figure 1. A comparison of DART reconstructions using SIRT and TV as initial solutions using all of the projections in a data set, in this case 73 images. The top images show the initial solutions, while the bottom images show slices through the final reconstruction. The graph displays the relative error at every iteration of both methods.
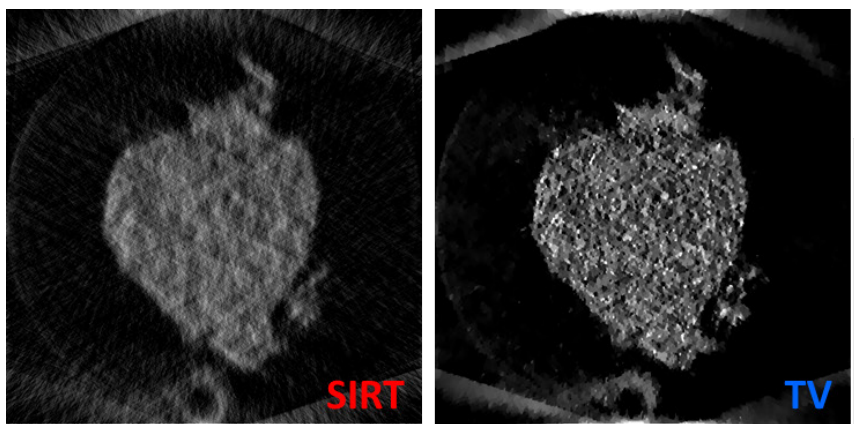

\section{Projections}
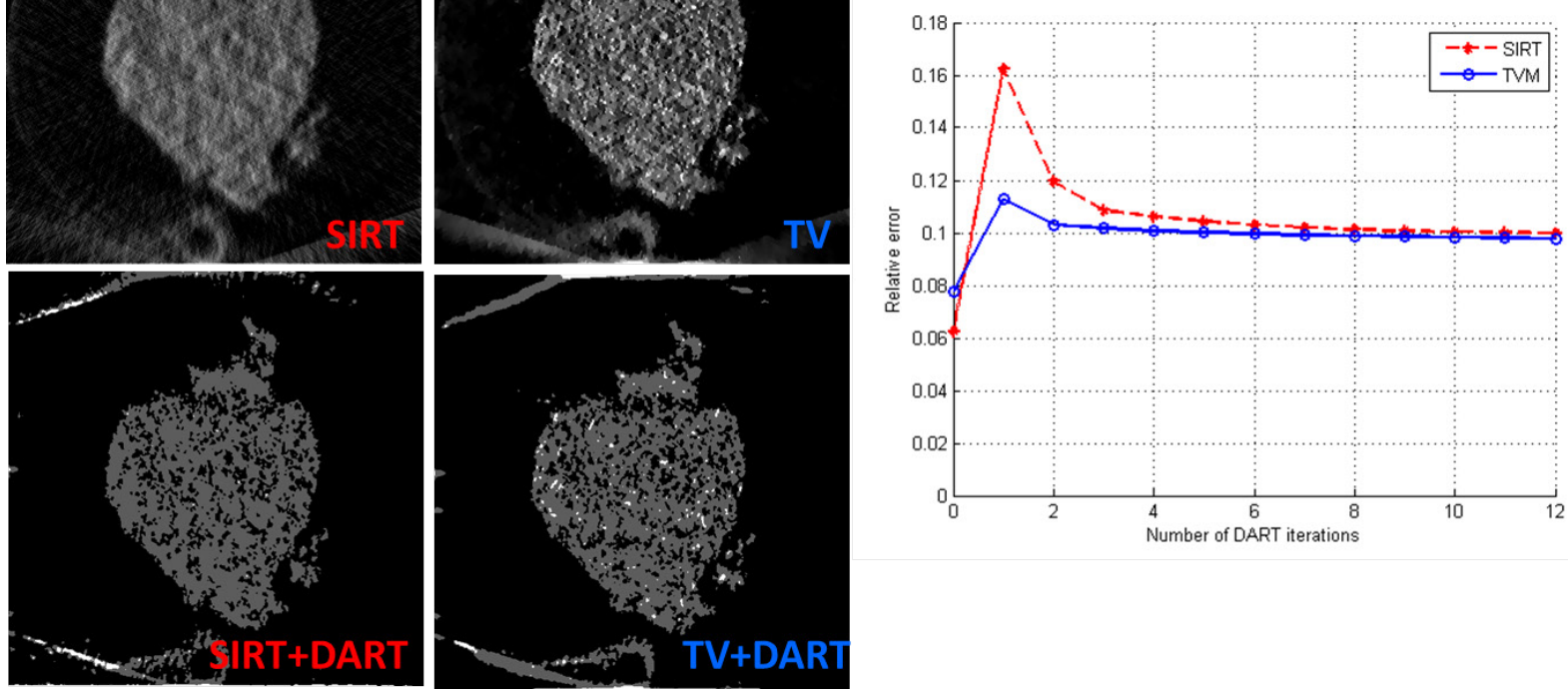

Figure 2. A comparison of DART reconstructions using SIRT and TV as initial solutions using half of the projections in a data set, in this case 37 images. The top images show the initial solutions, while the bottom images show slices through the final reconstruction. The graph displays the relative error at every iteration of both methods. 